Proyecciones Journal of Mathematics

Vol. 37, $\mathrm{N}^{\circ}$ 4, pp. 731-747, December 2018.

Universidad Católica del Norte

Antofagasta - Chile

\title{
Dual third-order Jacobsthal quaternions
}

\author{
Gamaliel Cerda-Morales \\ Pontificia Universidad Católica de Valparaíso, Chile \\ Received : February 2018. Accepted : April 2018
}

\begin{abstract}
In 2016, Yüce and Torunbalcı Aydın [18] defined dual Fibonacci quaternions. In this paper, we defined the dual third-order Jacobsthal quaternions and dual third-order Jacobsthal-Lucas quaternions. Also, we investigated the relations between the dual third-order Jacobsthal quaternions and third-order Jacobsthal numbers. Furthermore, we gave some their quadratic properties, the summations, the Binet's formulas and Cassini-like identities for these quaternions.
\end{abstract}

Subjclass Mathematical subject classification : Primary: 11R52; Secondary: 11B37, 20G20.

Key words : Third-order Jacobsthal number, third-order JacobsthalLucas number, third-order Jacobsthal quaternions, third-order JacobsthalLucas quaternions, dual quaternion. 


\section{Introduction}

The real quaternions are a number system which extends to the complex numbers. They are first described by Irish mathematician William Rowan Hamilton in 1843. In 1963, Horadam [9] defined the $n$-th Fibonacci quaternion which can be represented as

$Q_{F}=\left\{Q_{n}=F_{n}+\mathbf{i} F_{n+1}+\mathbf{j} F_{n+2}+\mathbf{k} F_{n+3}: F_{n}\right.$ is $n-t h$ Fibonacci number $\}$, (1.1)

where $\mathbf{i}^{2}=\mathbf{j}^{2}=\mathbf{k}^{2}=\mathbf{i j k}=-1$.

In 1969, Iyer $[14,15]$ derived many relations for the Fibonacci quaternions. In 1977, Iakin $[12,13]$ introduced higher order quaternions and gave some identities for these quaternions. Furthermore, Horadam [10] extend to quaternions to the complex Fibonacci numbers defined by Harman [6]. In 2012, Halıcı [6] gave generating functions and Binet's formulas for Fibonacci and Lucas quaternions.

In 2006, Majernik [16] defined a new type of quaternions, the so-called dual quaternions in the form $Q_{\mathbf{N}}=\{a+b \mathbf{i}+c \mathbf{j}+d \mathbf{k}: a, b, c, d \in \mathbf{R}\}$, with the following multiplication schema for the quaternion units

$$
\mathbf{i}^{2}=\mathbf{j}^{2}=\mathbf{k}^{2}=0, \mathbf{i j}=-\mathbf{j i}=\mathbf{j} \mathbf{k}=-\mathbf{k j}=\mathbf{k i}=-\mathbf{i k}=0 .
$$

In 2009, Ata and Yaylı [1] defined dual quaternions with dual numbers coefficient as follows:

$Q_{\mathbf{D}}=\left\{A+B \mathbf{i}+C \mathbf{j}+D \mathbf{k}: A, B, C, D \in \mathbf{D}, \mathbf{i}^{2}=\mathbf{j}^{2}=\mathbf{k}^{2}=\mathbf{i j} \mathbf{k}=-1\right\}$, (1.3)

where $\mathbf{D}=\mathbf{R}[\varepsilon]=\left\{a+b \varepsilon: a, b \in \mathbf{R}, \varepsilon^{2}=0, \varepsilon \neq 0\right\}$. It is clear that $Q_{\mathbf{N}}$ and $Q_{\mathbf{D}}$ are different sets. In 2014, Nurkan and Güven [17] defined dual Fibonacci quaternions as follows:

(1.4) $\mathbf{D}_{F}=\left\{Q_{n}=\widehat{F}_{n}+\mathbf{i} \widehat{F}_{n+1}+\mathbf{j} \widehat{F}_{n+2}+\mathbf{k} \widehat{F}_{n+3}: \widehat{F}_{n}=F_{n}+\varepsilon F_{n+1}\right\}$,

where $\mathbf{i}^{2}=\mathbf{j}^{2}=\mathbf{k}^{2}=\mathbf{i j k}=-1$ and $\widehat{F}_{n}$ is the $n$-th dual Fibonacci number.

In 2016, Yüce and Torunbalcı Aydın [18] defined dual Fibonacci quaternions as follows: 


$$
\mathbf{N}_{F}=\left\{Q_{n}=F_{n}+\mathbf{i} F_{n+1}+\mathbf{j} F_{n+2}+\mathbf{k} F_{n+3}:\right.
$$

$\mathrm{F}_{n}$ is $n$-th Fibonacci number $\}$,

where $\mathbf{i}^{2}=\mathbf{j}^{2}=\mathbf{k}^{2}=0, \mathbf{i} \mathbf{j}=-\mathbf{j i}=\mathbf{j} \mathbf{k}=-\mathbf{k j}=\mathbf{k} \mathbf{i}=-\mathbf{i} \mathbf{k}=0$. For more details on dual quaternions and generalized dual Fibonacci quaternions, see $[5,19]$.

On the other hand, the Jacobsthal numbers have many interesting properties and applications in many fields of science (see, e.g., [2]). The Jacobsthal numbers $J_{n}$ are defined by the recurrence relation

$$
J_{0}=0, J_{1}=1, J_{n+1}=J_{n}+2 J_{n-1}, n \geq 1 .
$$

Another important sequence is the Jacobsthal-Lucas sequence. This sequence is defined by the recurrence relation $j_{n+1}=j_{n}+2 j_{n-1}, n \geq 1$ and $j_{0}=2, j_{1}=1$. (see, [11]).

In [4], the Jacobsthal recurrence relation (1.6) is extended to higher order recurrence relations and the basic list of identities provided by A. F. Horadam [11] is expanded and extended to several identities for some of the higher order cases. In particular, third-order Jacobsthal numbers, $\left\{J_{n}^{(3)}\right\}_{n \geq 0}$, and third-order Jacobsthal-Lucas numbers, $\left\{j_{n}^{(3)}\right\}_{n \geq 0}$, are defined by

$$
J_{n+3}^{(3)}=J_{n+2}^{(3)}+J_{n+1}^{(3)}+2 J_{n}^{(3)}, J_{0}^{(3)}=0, J_{1}^{(3)}=J_{2}^{(3)}=1, n \geq 0,
$$

and

$$
j_{n+3}^{(3)}=j_{n+2}^{(3)}+j_{n+1}^{(3)}+2 j_{n}^{(3)}, j_{0}^{(3)}=2, j_{1}^{(3)}=1, j_{2}^{(3)}=5, n \geq 0,
$$

respectively.

The following properties given for third order Jacobsthal numbers and third order Jacobsthal-Lucas numbers play important roles in this paper (for more, see $[3,4]$ ).

$$
3 J_{n}^{(3)}+j_{n}^{(3)}=2^{n+1},
$$




$$
\begin{aligned}
& j_{n}^{(3)}-3 J_{n}^{(3)}=2 j_{n-3}^{(3)}, \\
& J_{n+2}^{(3)}-4 J_{n}^{(3)}=\left\{\begin{array}{ccc}
-2 & \text { if } & n \equiv 1(\bmod 3) \\
1 & \text { if } & n \neq \equiv 1(\bmod 3)
\end{array},\right. \\
& j_{n}^{(3)}-4 J_{n}^{(3)}=\left\{\begin{array}{ccc}
2 & \text { if } & n \equiv 0(\bmod 3) \\
-3 & \text { if } & n \equiv 1(\bmod 3) \\
1 & \text { if } & n \equiv 2(\bmod 3)
\end{array},\right. \\
& j_{n+1}^{(3)}+j_{n}^{(3)}=3 J_{n+2}^{(3)}, \\
& j_{n}^{(3)}-J_{n+2}^{(3)}=\left\{\begin{array}{cll}
1 & \text { if } & n \equiv 0(\bmod 3) \\
-1 & \text { if } & n \equiv 1(\bmod 3) \\
0 & \text { if } & n \equiv 2(\bmod 3)
\end{array},\right. \\
& \left(j_{n-3}^{(3)}\right)^{2}+3 J_{n}^{(3)} j_{n}^{(3)}=4^{n}, \\
& \sum_{k=0}^{n} J_{k}^{(3)}=\left\{\begin{array}{ccc}
J_{n+1}^{(3)} & \text { if } & n \neq \equiv(\bmod 3) \\
J_{n+1}^{(3)}-1 & \text { if } & n \equiv 0(\bmod 3)
\end{array}\right.
\end{aligned}
$$

and

$$
\left(j_{n}^{(3)}\right)^{2}-9\left(J_{n}^{(3)}\right)^{2}=2^{n+2} j_{n-3}^{(3)} .
$$

Using standard techniques for solving recurrence relations, the auxiliary equation, and its roots are given by

$$
x^{3}-x^{2}-x-2=0 ; x=2, \text { and } x=\frac{-1 \pm i \sqrt{3}}{2} .
$$

Note that the latter two are the complex conjugate cube roots of unity. Call them $\omega_{1}$ and $\omega_{2}$, respectively. Thus the Binet formulas can be written as

$(1.18) J_{n}^{(3)}=\frac{1}{7} 2^{n+1}-\frac{3+2 i \sqrt{3}}{21} \omega_{1}^{n}-\frac{3-2 i \sqrt{3}}{21} \omega_{2}^{n}=\frac{1}{7}\left(2^{n+1}-V_{n}^{(3)}\right)$

and 
$j_{n}^{(3)}=\frac{1}{7} 2^{n+3}+\frac{3+2 i \sqrt{3}}{7} \omega_{1}^{n}+\frac{3-2 i \sqrt{3}}{7} \omega_{2}^{n}=\frac{1}{7}\left(2^{n+3}+3 V_{n}^{(3)}\right)$,

respectively. Here $V_{n}^{(3)}$ is the sequence defined by

$V_{n}^{(3)}=\frac{3+2 i \sqrt{3}}{3} \omega_{1}^{n}+\frac{3-2 i \sqrt{3}}{3} \omega_{2}^{n}=\left\{\begin{array}{ccc}2 & \text { if } & n \equiv 0(\bmod 3) \\ -3 & \text { if } & n \equiv 1(\bmod 3) \\ 1 & \text { if } & n \equiv 2(\bmod 3)\end{array}\right.$.

Using Eq. (1.20) is easy to see that for all $n \geq 0$ :

$$
V_{n}^{(3)}+2 V_{n+1}^{(3)}+4 V_{n+2}^{(3)}=\left\{\begin{array}{ccc}
0 & \text { if } & n \equiv 0(\bmod 3) \\
7 & \text { if } & n \equiv 1(\bmod 3) \\
-7 & \text { if } & n \equiv 2(\bmod 3)
\end{array} .\right.
$$

Recently in [3], we have defined a new type of quaternions with the third-order Jacobsthal and third-order Jacobsthal-Lucas number components as

$$
J Q_{n}^{(3)}=J_{n}^{(3)}+J_{n+1}^{(3)} \mathbf{i}+J_{n+2}^{(3)} \mathbf{j}+J_{n+3}^{(3)} \mathbf{k}
$$

and

$$
j Q_{n}^{(3)}=j_{n}^{(3)}+j_{n+1}^{(3)} \mathbf{i}+j_{n+2}^{(3)} \mathbf{j}+j_{n+3}^{(3)} \mathbf{k},
$$

respectively, where $\mathbf{i}^{2}=\mathbf{j}^{2}=\mathbf{k}^{2}=\mathbf{i j k}=-1$, and we studied the properties of these quaternions. Also, we derived the generating functions and many other identities for the third-order Jacobsthal and third-order JacobsthalLucas quaternions.

In this paper, we define the dual third-order Jacobsthal quaternions and dual third-order Jacobsthal-Lucas quaternions as follows:

$$
J N_{m}^{(3)}=J_{m}^{(3)}+J_{m+1}^{(3)} \mathbf{i}+J_{m+2}^{(3)} \mathbf{j}+J_{m+3}^{(3)} \mathbf{k}(m \geq 0)
$$

and

$$
j N_{m}^{(3)}=j_{m}^{(3)}+j_{m+1}^{(3)} \mathbf{i}+j_{m+2}^{(3)} \mathbf{j}+j_{m+3}^{(3)} \mathbf{k}(m \geq 0),
$$


respectively. Here $\mathbf{i}^{2}=\mathbf{j}^{2}=\mathbf{k}^{2}=0, \mathbf{i j}=-\mathbf{j i}=\mathbf{j} \mathbf{k}=-\mathbf{k j}=\mathbf{k} \mathbf{i}=$ $-\mathbf{i k}=0$. Also, we investigated the relations between the dual third-order Jacobsthal quaternions and third-order Jacobsthal numbers. Furthermore, we give some their quadratic properties, the Binet's formulas, d'Ocagne and Cassini-like identities for these quaternions.

\section{Dual Third-Order Jacobsthal Quaternions}

We can define dual third-order Jacobsthal quaternions by using third-order Jacobsthal numbers. The $n$-th third-order Jacobsthal number $J_{n}^{(3)}$ is defined by Eq. (1.7). Then, we can define the dual third-order Jacobsthal quaternions as follows:

$$
\mathbf{N}_{J}=\left\{J N_{m}^{(3)}=J_{m}^{(3)}+J_{m+1}^{(3)} \mathbf{i}+J_{m+2}^{(3)} \mathbf{j}+J_{m+3}^{(3)} \mathbf{k}: m 0\right\}
$$

where $J_{m}^{(3)}$ is the $m$-th third-order Jacobsthal number and $\{\mathbf{i}, \mathbf{j}, \mathbf{k}\}$ as in Eq. (1.2). Also, we can define the dual third-order Jacobsthal-Lucas quaternion as follows:

$$
\mathbf{N}_{j}=\left\{j N_{m}^{(3)}=j_{m}^{(3)}+j_{m+1}^{(3)} \mathbf{i}+j_{m+2}^{(3)} \mathbf{j}+j_{m+3}^{(3)} \mathbf{k}: m 0\right\}
$$

where $j_{m}^{(3)}$ is the $m$-th third-order Jacobsthal-Lucas number.

Then, the addition and subtraction of the dual third-order Jacobsthal and dual third-order Jacobsthal-Lucas quaternions is defined by

$$
\begin{aligned}
& J N_{m}^{(3)} \pm j N_{m}^{(3)} \\
& =\left(J_{m}^{(3)}+J_{m+1}^{(3)} \mathbf{i}+J_{m+2}^{(3)} \mathbf{j}+J_{m+3}^{(3)} \mathbf{k}\right) \\
& \pm\left(j_{m}^{(3)}+j_{m+1}^{(3)} \mathbf{i}+j_{m+2}^{(3)} \mathbf{j}+j_{m+3}^{(3)} \mathbf{k}\right) \\
& =\left(J_{m}^{(3)} \pm j_{m}^{(3)}\right)+\left(J_{m+1}^{(3)} \pm j_{m+1}^{(3)}\right) \mathbf{i}+\left(J_{m+2}^{(3)} \pm j_{m+2}^{(3)}\right) \mathbf{j} \\
& +\left(J_{m+3}^{(3)} \pm j_{m+3}^{(3)}\right) \mathbf{k}
\end{aligned}
$$

and the multiplication of the dual third-order Jacobsthal and dual thirdorder Jacobsthal-Lucas quaternions is defined by 


$$
\begin{aligned}
& J N_{m}^{(3)} j N_{m}^{(3)} \\
(2.4) & =\left(J_{m}^{(3)}+J_{m+1}^{(3)} \mathbf{i}+J_{m+2}^{(3)} \mathbf{j}+J_{m+3}^{(3)} \mathbf{k}\right)\left(j_{m}^{(3)}+j_{m+1}^{(3)} \mathbf{i}+j_{m+2}^{(3)} \mathbf{j}+j_{m+3}^{(3)} \mathbf{k}\right) \\
= & J_{m}^{(3)} j_{m}^{(3)}+\left(J_{m}^{(3)} j_{m+1}^{(3)}+J_{m+1}^{(3)} j_{m}^{(3)}\right) \mathbf{i}+\left(J_{m}^{(3)} j_{m+2}^{(3)}+J_{m+2}^{(3)} j_{m}^{(3)}\right) \mathbf{j} \\
& +\left(J_{m}^{(3)} j_{m+3}^{(3)}+J_{m+3}^{(3)} j_{m}^{(3)}\right) \mathbf{k} .
\end{aligned}
$$

Now, the scalar and the vector part of the $J N_{m}^{(3)}$ which is the $m$-th term of the dual third-order Jacobsthal sequence $\left\{J N_{m}^{(3)}\right\}_{m \geq 0}$ are denoted by

$$
\left(S_{J N_{m}^{(3)}}, V_{J N_{m}^{(3)}}\right)=\left(J_{m}^{(3)}, J_{m+1}^{(3)} \mathbf{i}+J_{m+2}^{(3)} \mathbf{j}+J_{m+3}^{(3)} \mathbf{k}\right) .
$$

Thus, the dual third-order Jacobsthal $J N_{m}^{(3)}$ is given by $S_{J N_{m}^{(3)}}+V_{J N_{m}^{(3)}}$. Then, relation (2.4) is defined by

$$
J N_{m}^{(3)} j N_{m}^{(3)}=S_{J N_{m}^{(3)}} S_{j N_{m}^{(3)}}+S_{J N_{m}^{(3)}} V_{j N_{m}^{(3)}}+S_{j N_{m}^{(3)}} V_{J N_{m}^{(3)}} .
$$

The conjugate of dual third-order Jacobsthal quaternion $J N_{m}^{(3)}$ is denoted by $\overline{J N}_{m}^{(3)}$ and it is $\overline{J N}_{m}^{(3)}=J_{m}^{(3)}-J_{m+1}^{(3)} \mathbf{i}-J_{m+2}^{(3)} \mathbf{j}-J_{m+3}^{(3)} \mathbf{k}$. The norm of $J N_{m}^{(3)}$ is defined as

$$
N r^{2}\left(J N_{m}^{(3)}\right)=J N_{m}^{(3)} \overline{J N}_{m}^{(3)}=\overline{J N}_{m}^{(3)} J N_{m}^{(3)}=\left(J N_{m}^{(3)}\right)^{2} .
$$

Then, we give the following theorem using statements (2.1), (2.3) and (2.4).

Theorem 2.1. Let $J_{m}^{(3)}$ and $J N_{m}^{(3)}$ be the $m$-th terms of the third-order Jacobsthal sequence $\left\{J_{m}^{(3)}\right\}_{m \geq 0}$ and the dual third-order Jacobsthal quaternion sequence $\left\{J N_{m}^{(3)}\right\}_{m \geq 0}$, respectively. In this case, for $m \geq 0$ we can give the following relations:

$$
\begin{gathered}
2 J N_{m}^{(3)}+J N_{m+1}^{(3)}+J N_{m+2}^{(3)}=J N_{m+3}^{(3)}, \\
J N_{m}^{(3)}-J N_{m+1}^{(3)} \mathbf{i}-J N_{m+2}^{(3)} \mathbf{j}-J N_{m+3}^{(3)} \mathbf{k}=J_{m}^{(3)},
\end{gathered}
$$


$\left(J N_{m}^{(3)}\right)^{2}+\left(J N_{m+1}^{(3)}\right)^{2}+\left(J N_{m+2}^{(3)}\right)^{2}=\frac{1}{7}\left(\begin{array}{c}3 \cdot 2^{2(m+1)}(1+4 \mathbf{i}+8 \mathbf{j}+16 \mathbf{k}) \\ -2^{m+2} U N_{m}^{(3)} \\ -2^{m+3} U_{m}^{(3)}(\mathbf{i}+2 \mathbf{j}+4 \mathbf{k}) \\ +2(1-\mathbf{i}-\mathbf{j}+2 \mathbf{k})\end{array}\right)$

where

$$
U N_{m}^{(3)}=U_{m}^{(3)}+U_{m+1}^{(3)} \mathbf{i}+U_{m+2}^{(3)} \mathbf{j}+U_{m+3}^{(3)} \mathbf{k} \text { and } U_{m}^{(3)}=\frac{1}{7}\left(V_{m+1}^{(3)}+3 V_{m+2}^{(3)}\right)
$$

Proof. (2.8): By the equations $J N_{m}^{(3)}=J_{m}^{(3)}+J_{m+1}^{(3)} \mathbf{i}+J_{m+2}^{(3)} \mathbf{j}+J_{m+3}^{(3)} \mathbf{k}$ and (1.7), we get $2 \mathrm{JN}_{m}^{(3)}+J N_{m+1}^{(3)}+J N_{m+2}^{(3)}$

$=\left(2 J_{m}^{(3)}+2 J_{m+1}^{(3)} \mathbf{i}+2 J_{m+2}^{(3)} \mathbf{j}+2 J_{m+3}^{(3)} \mathbf{k}\right)$

$+\left(J_{m+1}^{(3)}+J_{m+2}^{(3)} \mathbf{i}+J_{m+3}^{(3)} \mathbf{j}+J_{m+4}^{(3)} \mathbf{k}\right)$

$+\left(J_{m+2}^{(3)}+J_{m+3}^{(3)} \mathbf{i}+J_{m+4}^{(3)} \mathbf{j}+J_{m+5}^{(3)} \mathbf{k}\right)$

$=\left(2 J_{m}^{(3)}+J_{m+1}^{(3)}+J_{m+2}^{(3)}\right)+\left(2 J_{m+1}^{(3)}+J_{m+2}^{(3)}+J_{m+3}^{(3)}\right) \mathbf{i}$

$+\left(2 J_{m+2}^{(3)}+J_{m+3}^{(3)}+J_{m+4}^{(3)}\right) \mathbf{j}+\left(2 J_{m+3}^{(3)}+J_{m+4}^{(3)}+J_{m+5}^{(3)}\right) \mathbf{k}$

$=J_{m+3}^{(3)}+J_{m+4}^{(3)} \mathbf{i}+J_{m+5}^{(3)} \mathbf{j}+J_{m+6}^{(3)} \mathbf{k}$

$=J N_{m+3}^{(3)}$.

(2.9): By using $J N_{m}^{(3)}$ in the Eq. (2.1) and conditions (1.2), we get

$$
\begin{aligned}
& \mathrm{JN}_{m}^{(3)}-J N_{m+1}^{(3)} \mathbf{i}-J N_{m+2}^{(3)} \mathbf{j}-J N_{m+3}^{(3)} \mathbf{k} \\
= & J_{m}^{(3)}+J_{m+1}^{(3)} \mathbf{i}+J_{m+2}^{(3)} \mathbf{j}+J_{m+3}^{(3)} \mathbf{k} \\
- & \left(J_{m+1}^{(3)}+J_{m+2}^{(3)} \mathbf{i}+J_{m+3}^{(3)} \mathbf{j}+J_{m+4}^{(3)} \mathbf{k}\right) \mathbf{i} \\
- & \left(J_{m+2}^{(3)}+J_{m+3}^{(3)} \mathbf{i}+J_{m+4}^{(3)} \mathbf{j}+J_{m+5}^{(3)} \mathbf{k}\right) \mathbf{j} \\
- & \left(J_{m+3}^{(3)}+J_{m+4}^{(3)} \mathbf{i}+J_{m+5}^{(3)} \mathbf{j}+J_{m+6}^{(3)} \mathbf{k}\right) \mathbf{k} \\
= & J_{m}^{(3)} .
\end{aligned}
$$

(2.10): By using Eqs. (2.4) and (1.18), we get

$$
\left(J N_{m}^{(3)}\right)^{2}=\left(J_{m}^{(3)}\right)^{2}+2 J_{m}^{(3)} J_{m+1}^{(3)} \mathbf{i}+2 J_{m}^{(3)} J_{m+2}^{(3)} \mathbf{j}+2 J_{m}^{(3)} J_{m+3}^{(3)} \mathbf{k}
$$

and 


$$
\begin{aligned}
& \left(J_{m}^{(3)}\right)^{2} \\
& +\left(J_{m+1}^{(3)}\right)^{2}+\left(J_{m+2}^{(3)}\right)^{2} \\
(2.12) & =\frac{1}{49}\left(\left(2^{m+1}-V_{m}^{(3)}\right)^{2}+\left(2^{m+2}-V_{m+1}^{(3)}\right)^{2}+\left(2^{m+3}-V_{m+2}^{(3)}\right)^{2}\right) \\
& =\frac{1}{49}\left(21 \cdot 2^{2(m+1)}-2^{m+2}\left(V_{m}^{(3)}+2 V_{m+1}^{(3)}+4 V_{m+2}^{(3)}\right)+14\right) \\
& =\frac{1}{7}\left(3 \cdot 2^{2(m+1)}-2^{m+2} U_{m}^{(3)}+2\right),
\end{aligned}
$$

where $U_{m}^{(3)}=\frac{1}{7}\left(V_{m}^{(3)}+2 V_{m+1}^{(3)}+4 V_{m+2}^{(3)}\right)=\frac{1}{7}\left(V_{m+1}^{(3)}+3 V_{m+2}^{(3)}\right)$. Finally, from the Eqs. (2.11) and (2.12), we obtain

$$
\begin{aligned}
& \left(J N_{m}^{(3)}\right)^{2}+\left(J N_{m+1}^{(3)}\right)^{2}+\left(J N_{m+2}^{(3)}\right)^{2} \\
= & \left(J_{m}^{(3)}\right)^{2}+\left(J_{m+1}^{(3)}\right)^{2}+\left(J_{m+2}^{(3)}\right)^{2} \\
+ & 2\left(J_{m}^{(3)} J_{m+1}^{(3)}+J_{m+1}^{(3)} J_{m+2}^{(3)}+J_{m+2}^{(3)} J_{m+3}^{(3)}\right) \mathbf{i} \\
+ & 2\left(J_{m}^{(3)} J_{m+2}^{(3)}+J_{m+1}^{(3)} J_{m+3}^{(3)}+J_{m+2}^{(3)} J_{m+4}^{(3)}\right) \mathbf{j} \\
+ & 2\left(J_{m}^{(3)} J_{m+3}^{(3)}+J_{m+1}^{(3)} J_{m+4}^{(3)}+J_{m+2}^{(3)} J_{m+5}^{(3)}\right) \mathbf{k} \\
= & \frac{1}{7}\left(\begin{array}{c}
3 \cdot 2^{2(m+1)}(1+4 \mathbf{i}+8 \mathbf{j}+16 \mathbf{k})-2^{m+2} U N_{m}^{(3)} \\
-2^{m+3} U_{m}^{(3)}(\mathbf{i}+2 \mathbf{j}+4 \mathbf{k})+2(1-\mathbf{i}-\mathbf{j}+2 \mathbf{k})
\end{array}\right),
\end{aligned}
$$

where $U N_{m}^{(3)}=U_{m}^{(3)}+U_{m+1}^{(3)} \mathbf{i}+U_{m+2}^{(3)} \mathbf{j}+U_{m+3}^{(3)} \mathbf{k}$.

Theorem 2.2. Let $J N_{m}^{(3)}$ and $j N_{m}^{(3)}$ be the $m$-th terms of the dual thirdorder Jacobsthal quaternion sequence $\left\{J N_{m}^{(3)}\right\}_{m \geq 0}$ and the dual third-order Jacobsthal-Lucas quaternion sequence $\left\{j N_{m}^{(3)}\right\}_{m \geq 0}$, respectively. The following relations are satisfied

$$
\begin{gathered}
j N_{m+3}^{(3)}-3 J N_{m+3}^{(3)}=2 j N_{m}^{(3)}, \\
j N_{m+1}^{(3)}+j N_{m}^{(3)}=3 J N_{m+2}^{(3)}, \\
\left(j N_{m}^{(3)}\right)^{2}+3 J N_{m+3}^{(3)} j N_{m+3}^{(3)}=4^{m+3}(1+4 \mathbf{i}+8 \mathbf{j}+16 \mathbf{k}) .
\end{gathered}
$$


Proof. (2.13): From identities between third-order Jacobsthal number and third-order Jacobsthal-Lucas number (1.10) and (2.3), it follows that

$$
\begin{aligned}
& \mathrm{jN}_{m+3}^{(3)}-3 J N_{m+3}^{(3)}=j_{m+3}^{(3)}+j_{m+4}^{(3)} \mathbf{i}+j_{m+5}^{(3)} \mathbf{j}+j_{m+6}^{(3)} \mathbf{k} \\
- & 3\left(J_{m+3}^{(3)}+J_{m+4}^{(3)} \mathbf{i}+J_{m+5}^{(3)} \mathbf{j}+J_{m+6}^{(3)} \mathbf{k}\right) \\
= & \left(j_{m+3}^{(3)}-3 J_{m+3}^{(3)}\right)+\left(j_{m+4}^{(3)}-3 J_{m+4}^{(3)}\right) \mathbf{i} \\
+ & \left(j_{m+5}^{(3)}-3 J_{m+5}^{(3)} \mathbf{j}+\left(j_{m+6}^{(3)}-3 J_{m+6}^{(3)}\right) \mathbf{k}\right. \\
= & 2 j_{m}^{(3)}+2 j_{m+1}^{(3)} \mathbf{i}+2 j_{m+2}^{(3)} \mathbf{j}+2 j_{m+3}^{(3)} \mathbf{k} \\
= & 2 j N_{m}^{(3)} .
\end{aligned}
$$

The proof of (2.14) is similar to (2.13), using the identity (1.13). (2.15):

Now, using Eqs. $(2.4),(2.11)$ and $(1.15)$, we get $\left(j N_{m}^{(3)}\right)^{2}+3 J N_{m+3}^{(3)} j N_{m+3}^{(3)}$

$$
\begin{aligned}
& =\left(j_{m}^{(3)}\right)^{2}+2 j_{m}^{(3)} j_{m+1}^{(3)} \mathbf{i}+2 j_{m}^{(3)} j_{m+2}^{(3)} \mathbf{j}+2 j_{m}^{(3)} j_{m+3}^{(3)} \mathbf{k} \\
& +3 J_{m+3}^{(3)} j_{m+3}^{(3)}+3\left(J_{m+3}^{(3)} j_{m+4}^{(3)}+J_{m+4}^{(3)} j_{m+3}^{(3)}\right) \mathbf{i} \\
& +3\left(J_{m+3}^{(3)} j_{m+5}^{(3)}+J_{m+5}^{(3)} j_{m+3}^{(3)} \mathbf{j}+3\left(J_{m+3}^{(3)} j_{m+6}^{(3)}+J_{m+6}^{(3)} j_{m+3}^{(3)}\right) \mathbf{k}\right. \\
& =\left(j_{m}^{(3)}\right)^{2}+3 J_{m+3}^{(3)} j_{m+3}^{(3)}+\left(2 j_{m}^{(3)} j_{m+1}^{(3)}+3\left(J_{m+3}^{(3)} j_{m+4}^{(3)}+J_{m+4}^{(3)} j_{m+3}^{(3)}\right)\right) \mathbf{i} \\
& +\left(2 j_{m}^{(3)} j_{m+2}^{(3)}+3\left(J_{m+3}^{(3)} j_{m+5}^{(3)}+J_{m+5}^{(3)} j_{m+3}^{(3)}\right)\right) \mathbf{j} \\
& +\left(2 j_{m}^{(3)} j_{m+3}^{(3)}+3\left(J_{m+3}^{(3)} j_{m+6}^{(3)}+J_{m+6}^{(3)} j_{m+3}^{(3)}\right)\right) \mathbf{k} \\
& =4^{m+3}(1+4 \mathbf{i}+8 \mathbf{j}+16 \mathbf{k}),
\end{aligned}
$$

the last equality because $3 J_{m+3}^{(3)} j_{m+3}^{(3)}=4^{m+3}-\left(j_{m}^{(3)}\right)^{2}$ in Eq. (1.15).

Theorem 2.3. Let $J N_{m}^{(3)}$ be the $m$-th term of the dual third-order Jacobsthal quaternion sequence $\left\{J N_{m}^{(3)}\right\}_{m \geq 0}$. Then, we have the following identity

$\sum_{s=0}^{m} J N_{s}^{(3)}=J N_{m+1}^{(3)}-\frac{1}{21}\left(7(1+\mathbf{i}+4 \mathbf{j}+7 \mathbf{k})-4 V N_{m+1}^{(3)}+V N_{m}^{(3)}\right)$,

where $V N_{m}^{(3)}=V_{m}^{(3)}+V_{m+1}^{(3)} \mathbf{i}+V_{m+2}^{(3)} \mathbf{j}+V_{m+3}^{(3)} \mathbf{k}$.

Proof. Since

$\sum_{s=0}^{m} J_{s}^{(3)}=J_{m+1}^{(3)}-\frac{1}{21}\left(7-4 V_{m+1}^{(3)}+V_{m}^{(3)}\right)=\left\{\begin{array}{cll}J_{m+1}^{(3)} & \text { if } & n \neq \equiv(\bmod 3) \\ J_{m+1}^{(3)}-1 & \text { if } & n \equiv 0(\bmod 3)\end{array}\right.$ 
(see [3]), we get

$$
\begin{aligned}
& \sum_{s=0}^{m} J N_{s}^{(3)}=\sum_{s=0}^{m} J_{s}^{(3)}+\mathbf{i} \sum_{s=1}^{m+1} J_{s}^{(3)}+\mathbf{j} \sum_{s=2}^{m+2} J_{s}^{(3)}+\mathbf{k} \sum_{s=3}^{m+3} J_{s}^{(3)} \\
&= J_{m+1}^{(3)}-\frac{1}{21}\left(7-4 V_{m+1}^{(3)}+V_{m}^{(3)}\right) \\
&+\left(J_{m+2}^{(3)}-\frac{1}{21}\left(7-4 V_{m+2}^{(3)}+V_{m+1}^{(3)}\right)\right) \mathbf{i} \\
&+\left(J_{m+3}^{(3)}-\frac{1}{21}\left(28-4 V_{m+3}^{(3)}+V_{m+2}^{(3)}\right)\right) \mathbf{j} \\
&+\left(J_{m+4}^{(3)}-\frac{1}{21}\left(49-4 V_{m+4}^{(3)}+V_{m+3}^{(3)}\right)\right) \mathbf{k} \\
&= J N_{m+1}^{(3)}-\frac{1}{21}\left(7(1+\mathbf{i}+4 \mathbf{j}+7 \mathbf{k})-4 V N_{m+1}^{(3)}+V N_{m}^{(3)}\right) \\
& \text { where } V N_{m}^{(3)}=V_{m}^{(3)}+V_{m+1}^{(3)} \mathbf{i}+V_{m+2}^{(3)} \mathbf{j}+V_{m+3}^{(3)} \mathbf{k} .
\end{aligned}
$$

Theorem 2.4. Let $J N_{m}^{(3)}$ and $j N_{m}^{(3)}$ be the $m$-th terms of the dual thirdorder Jacobsthal quaternion sequence $\left\{J N_{m}^{(3)}\right\}_{m \geq 0}$ and the dual third-order Jacobsthal-Lucas quaternion sequence $\left\{j N_{m}^{(3)}\right\}_{m \geq 0}$, respectively. Then, we have

$$
\begin{aligned}
& j N_{m}^{(3)} \overline{J N_{m}^{(3)}}-\overline{j N_{m}^{(3)}} J N_{m}^{(3)}=2\left(J_{m}^{(3)} j N_{m}^{(3)}-j_{m}^{(3)} J N_{m}^{(3)}\right), \\
& j N_{m}^{(3)} J N_{m}^{(3)}+\overline{j N}_{m}^{(3)} \overline{J N}_{m}^{(3)}=2 j_{m}^{(3)} J_{m}^{(3)} .
\end{aligned}
$$

Proof. (2.18): By the Eqs. (2.1), (2.2) and $\overline{J N}_{m}^{(3)}=J_{m}^{(3)}-J_{m+1}^{(3)} \mathbf{i}-$ $J_{m+2}^{(3)} \mathbf{j}-J_{m+3}^{(3)} \mathbf{k}$, we get $\mathbf{j} \mathrm{N}_{m}^{(3)} \overline{J N}_{m}^{(3)}-\overline{j N}_{m}^{(3)} J N_{m}^{(3)}$

$=\left(j_{m}^{(3)}+j_{m+1}^{(3)} \mathbf{i}+j_{m+2}^{(3)} \mathbf{j}+j_{m+3}^{(3)} \mathbf{k}\right)\left(J_{m}^{(3)}-J_{m+1}^{(3)} \mathbf{i}-J_{m+2}^{(3)} \mathbf{j}-J_{m+3}^{(3)} \mathbf{k}\right)$

$-\left(j_{m}^{(3)}-j_{m+1}^{(3)} \mathbf{i}-j_{m+2}^{(3)} \mathbf{j}-j_{m+3}^{(3)} \mathbf{k}\right)\left(J_{m}^{(3)}+J_{m+1}^{(3)} \mathbf{i}+J_{m+2}^{(3)} \mathbf{j}+J_{m+3}^{(3)} \mathbf{k}\right)$

$=2 J_{m}^{(3)}\left(j_{m+1}^{(3)} \mathbf{i}+j_{m+2}^{(3)} \mathbf{j}+j_{m+3}^{(3)} \mathbf{k}\right)-2 j_{m}^{(3)}\left(J_{m+1}^{(3)} \mathbf{i}+J_{m+2}^{(3)} \mathbf{j}+J_{m+3}^{(3)} \mathbf{k}\right)$ $=2\left(J_{m}^{(3)} j N_{m}^{(3)}-j_{m}^{(3)} J N_{m}^{(3)}\right)$.

$$
\begin{aligned}
& (2.19): \mathrm{jN}_{m}^{(3)} J N_{m}^{(3)}+\overline{j N}_{m}^{(3)} \overline{J N_{m}^{(3)}} \\
= & \left(j_{m}^{(3)}+j_{m+1}^{(3)} \mathbf{i}+j_{m+2}^{(3)} \mathbf{j}+j_{m+3}^{(3)} \mathbf{k}\right)\left(J_{m}^{(3)}+J_{m+1}^{(3)} \mathbf{i}+J_{m+2}^{(3)} \mathbf{j}+J_{m+3}^{(3)} \mathbf{k}\right) \\
+ & \left(j_{m}^{(3)}-j_{m+1}^{(3)} \mathbf{i}-j_{m+2}^{(3)} \mathbf{j}-j_{m+3}^{(3)} \mathbf{k}\right)\left(J_{m}^{(3)}-J_{m+1}^{(3)} \mathbf{i}-J_{m+2}^{(3)} \mathbf{j}-J_{m+3}^{(3)} \mathbf{k}\right) \\
= & j_{m}^{(3)} J_{m}^{(3)}+\left(j_{m}^{(3)} J_{m+1}^{(3)}+j_{m+1}^{(3)} J_{m}^{(3)}\right) \mathbf{i}+\left(j_{m}^{(3)} J_{m+2}^{(3)}+j_{m+2}^{(3)} J_{m}^{(3)}\right) \mathbf{j} \\
+ & \left(j_{m}^{(3)} J_{m+3}^{(3)}+j_{m+3}^{(3)} J_{m}^{(3)}\right) \mathbf{k} \\
+ & j_{m}^{(3)} J_{m}^{(3)}-\left(j_{m}^{(3)} J_{m+1}^{(3)}+j_{m+1}^{(3)} J_{m}^{(3)}\right) \mathbf{i}-\left(j_{m}^{(3)} J_{m+2}^{(3)}+j_{m+2}^{(3)} J_{m}^{(3)}\right) \mathbf{j} \\
- & \left(j_{m}^{(3)} J_{m+3}^{(3)}+j_{m+3}^{(3)} J_{m}^{(3)}\right) \mathbf{k} \\
= & 2 j_{m}^{(3)} J_{m}^{(3)} .
\end{aligned}
$$


Theorem 2.5 (Binet's Formulas). Let $J N_{m}^{(3)}$ and $j N_{m}^{(3)}$ be $m$-th terms of the dual third-order Jacobsthal quaternion sequence $\left\{J N_{m}^{(3)}\right\}_{m>0}$ and the dual third-order Jacobsthal-Lucas quaternion sequence $\left\{J N_{m}^{(3)}\right\}_{m \geq 0}$, respectively. For $m \geq 0$, the Binet's formulas for these quaternions are as follows:

$J N_{m}^{(3)}=\frac{1}{7} 2^{m+1} \underline{\alpha}-\frac{3+2 i \sqrt{3}}{21} \underline{\omega_{1}} \omega_{1}^{m}-\frac{3-2 i \sqrt{3}}{21} \underline{\omega_{2}} \omega_{2}^{m}=\frac{1}{7}\left(2^{m+1} \underline{\alpha}-V N_{m}^{(3)}\right)$

and

$j N_{m}^{(3)}=\frac{1}{7} 2^{m+3} \underline{\alpha}+\frac{3+2 i \sqrt{3}}{7} \underline{\omega_{1}} \omega_{1}^{m}+\frac{3-2 i \sqrt{3}}{7} \underline{\omega_{2}} \omega_{2}^{m}=\frac{1}{7}\left(2^{m+3} \underline{\alpha}+3 V N_{m}^{(3)}\right)$, $(2.21)$

respectively, where $V N_{m}^{(3)}$ is the sequence defined by

$$
V N_{m}^{(3)}=\left\{\begin{array}{ccc}
2-3 \mathbf{i}+\mathbf{j}+2 \mathbf{k} & \text { if } & n \equiv 0(\bmod 3) \\
-3+\mathbf{i}+2 \mathbf{j}-3 \mathbf{k} & \text { if } & n \equiv 1(\bmod 3) \\
1+2 \mathbf{i}-3 \mathbf{j}+\mathbf{k} & \text { if } & n \equiv 2(\bmod 3)
\end{array},\right.
$$

$\underline{\alpha}=1+2 \mathbf{i}+4 \mathbf{j}+8 \mathbf{k}$ and $\underline{\omega_{1,2}}=1+\omega_{1,2} \mathbf{i}+\omega_{1,2}^{2} \mathbf{j}+\mathbf{k}$.

Proof. Repeated use of (1.18) in (2.1) enables one to write for $\underline{\alpha}=$ $1+2 \mathbf{i}+4 \mathbf{j}+8 \mathbf{k}$ and $\omega_{1,2}=1+\omega_{1,2} \mathbf{i}+\omega_{1,2}^{2} \mathbf{j}+\mathbf{k}$,

$$
\begin{aligned}
& J N_{m}^{(3)} \\
& =J_{m}^{(3)}+J_{m+1}^{(3)} \mathbf{i}+J_{m+2}^{(3)} \mathbf{j}+J_{m+3}^{(3)} \mathbf{k} \\
& =\frac{1}{7} 2^{m+1}-\frac{3+2 i \sqrt{3}}{21} \omega_{1}^{m}-\frac{3-2 i \sqrt{3}}{21} \omega_{2}^{m} \\
& +\left(\frac{1}{7} 2^{m+2}-\frac{3+2 i \sqrt{3}}{21} \omega_{1}^{m+1}-\frac{3-2 i \sqrt{3}}{21} \omega_{2}^{m+1}\right) \mathbf{i} \\
& +\left(\frac{1}{7} 2^{m+3}-\frac{3+2 i \sqrt{3}}{21} \omega_{1}^{m+2}-\frac{3-2 i \sqrt{3}}{21} \omega_{2}^{m+2}\right) \mathbf{j} \\
& +\left(\frac{1}{7} 2^{m+4}-\frac{3+2 i \sqrt{3}}{21} \omega_{1}^{m+3}-\frac{3-2 i \sqrt{3}}{21} \omega_{2}^{m+3}\right) \mathbf{k} \\
& =\frac{1}{7} 2^{m+1} \underline{\alpha}+\frac{3+2 i \sqrt{3}}{7} \omega_{1} \omega_{1}^{m}+\frac{3-2 i \sqrt{3}}{7} \omega_{2} \omega_{2}^{m}
\end{aligned}
$$


and similarly making use of (1.19) in (2.2) yields

$j N_{m}^{(3)}=j_{m}^{(3)}+j_{m+1}^{(3)} \mathbf{i}+j_{m+2}^{(3)} \mathbf{j}+j_{m+3}^{(3)} \mathbf{k}=\frac{1}{7} 2^{m+3} \underline{\alpha}+\frac{3+2 i \sqrt{3}}{7} \underline{\omega_{1}} \omega_{1}^{m}+\frac{3-2 i \sqrt{3}}{7} \underline{\omega_{2}} \omega_{2}^{m}$.

The formulas in (2.23) and (2.24) are called as Binet's formulas for the dual third-order Jacobsthal and dual third-order Jacobsthal-Lucas quaternions, respectively. Using notation in (2.22), we obtain the results (2.20) and (2.21).

Theorem 2.6 (D'Ocagne-like Identity). Let $J N_{m}^{(3)}$ be the m-th terms of the dual third-order Jacobsthal quaternion sequence $\left\{J N_{m}^{(3)}\right\}_{m \geq 0}$. In this case, for $n \geq m \geq 0$, the d'Ocagne identities for $J N_{m}^{(3)}$ is as follows:

$J N_{n}^{(3)} J N_{m+1}^{(3)}-J N_{n+1}^{(3)} J N_{m}^{(3)}=\frac{1}{7}\left(\begin{array}{c}\underline{\alpha}\left(2^{n+1} U N_{m+1}^{(3)}-2^{m+1} U N_{n+1}^{(3)}\right) \\ +(1-\mathbf{i}-\mathbf{j}+2 \mathbf{k}) U_{n-m}^{(3)}\end{array}\right)$,

$$
\left(J N_{m+1}^{(3)}\right)^{2}-J N_{m+2}^{(3)} J N_{m}^{(3)}=\frac{1}{7}\left(\begin{array}{c}
2^{m+1} \underline{\alpha}\left(2 U N_{m+1}^{(3)}-U N_{m+2}^{(3)}\right) \\
+(1-\mathbf{i}-\mathbf{j}+2 \mathbf{k})
\end{array}\right),
$$

where $U N_{m+1}^{(3)}=\frac{1}{7}\left(2 V N_{m}^{(3)}-V N_{m+1}^{(3)}\right), \underline{\alpha}=1+2 \mathbf{i}+4 \mathbf{j}+8 \mathbf{k}$ and $U_{n}^{(3)}$ as in Eq. (2.12). 
Proof. (2.25): Using Eqs. (2.20) and (2.22), we get

$$
\begin{aligned}
& J N_{n}^{(3)} J N_{m+1}^{(3)}-J N_{n+1}^{(3)} J N_{m}^{(3)} \\
& =\frac{1}{49}\left(\begin{array}{c}
\left(2^{n+1} \underline{\alpha}-V N_{n}^{(3)}\right)\left(2^{m+2} \underline{\alpha}-V N_{m+1}^{(3)}\right) \\
-\left(2^{n+2} \underline{\alpha}-V N_{n+1}^{(3)}\right)\left(2^{m+1} \underline{\alpha}-V N_{m}^{(3)}\right)
\end{array}\right) \\
& =\frac{1}{49}\left(\begin{array}{c}
2^{n+m+3} \underline{\alpha}^{2}-2^{n+1} \underline{\alpha} V N_{m+1}^{(3)}-2^{m+2} V N_{n}^{(3)} \underline{\alpha}+V N_{n}^{(3)} V N_{m+1}^{(3)} \\
-2^{n+m+3} \underline{\alpha}^{2}+2^{n+2} \underline{\alpha} V N_{m}^{(3)}+2^{m+1} V N_{n+1}^{(3)} \underline{\alpha}-V N_{n+1}^{(3)} V N_{m}^{(3)}
\end{array}\right) \\
& =\frac{1}{7}\left(\begin{array}{c}
\underline{\alpha}\left(2^{n+1} U N_{m+1}^{(3)}-2^{m+1} U N_{n+1}^{(3)}\right)+(1-\mathbf{i}-\mathbf{j}+2 \mathbf{k}) U_{n-m}^{(3)}
\end{array}\right),
\end{aligned}
$$

where $U N_{m+1}^{(3)}=\frac{1}{7}\left(2 V N_{m}^{(3)}-V N_{m+1}^{(3)}\right)$ and $V N_{m}^{(3)}$ as in (2.22). In particular, if $n=m+1$ in Eq. (2.27), we obtain for $m \geq 0$,

$$
\left(J N_{m+1}^{(3)}\right)^{2}-J N_{m+2}^{(3)} J N_{m}^{(3)}=\frac{1}{7}\left(\begin{array}{c}
2^{m+1} \underline{\alpha}\left(2 U N_{m+1}^{(3)}-U N_{m+2}^{(3)}\right) \\
+(1-\mathbf{i}-\mathbf{j}+2 \mathbf{k})
\end{array}\right) .
$$

We will give an example in which we check in a particular case the Cassini-like identity for dual third-order Jacobsthal quaternions.

Example 2.7. Let $\left\{J N_{s}^{(3)}: s=0,1,2,3\right\}$ be the dual third-order Jacobsthal quaternions such that $J N_{0}^{(3)}=\mathbf{i}+\mathbf{j}+2 \mathbf{k}, J N_{1}^{(3)}=1+\mathbf{i}+2 \mathbf{j}+5 \mathbf{k}$, $J N_{2}^{(3)}=1+2 \mathbf{i}+5 \mathbf{j}+9 \mathbf{k}$ and $J N_{3}^{(3)}=2+5 \mathbf{i}+9 \mathbf{j}+18 \mathbf{k}$. In this case,

$$
\begin{aligned}
& \left(J N_{1}^{(3)}\right)^{2}-J N_{2}^{(3)} J N_{0}^{(3)} \\
& =(1+\mathbf{i}+2 \mathbf{j}+5 \mathbf{k})^{2}-(1+2 \mathbf{i}+5 \mathbf{j}+9 \mathbf{k})(\mathbf{i}+\mathbf{j}+2 \mathbf{k}) \\
& =(1+2 \mathbf{i}+4 \mathbf{j}+10 \mathbf{k})-(\mathbf{i}+\mathbf{j}+2 \mathbf{k}) \\
& =1+\mathbf{i}+3 \mathbf{j}+8 \mathbf{k} \\
& =\frac{1}{7}\left(\begin{array}{c}
2(1+2 \mathbf{i}+4 \mathbf{j}+8 \mathbf{k})\left(2 U N_{1}^{(3)}-U N_{2}^{(3)}\right) \\
+(1-\mathbf{i}-\mathbf{j}+2 \mathbf{k})
\end{array}\right)
\end{aligned}
$$


and

$$
\begin{aligned}
& \left(J N_{2}^{(3)}\right)^{2}-J N_{3}^{(3)} J N_{1}^{(3)} \\
& =(1+2 \mathbf{i}+5 \mathbf{j}+9 \mathbf{k})^{2}-(2+5 \mathbf{i}+9 \mathbf{j}+18 \mathbf{k})(1+\mathbf{i}+2 \mathbf{j}+5 \mathbf{k}) \\
& =(1+4 \mathbf{i}+10 \mathbf{j}+18 \mathbf{k})-(2+7 \mathbf{i}+13 \mathbf{j}+28 \mathbf{k}) \\
& =-1-3 \mathbf{i}-3 \mathbf{j}-10 \mathbf{k} \\
& =\frac{1}{7}\left(\begin{array}{c}
4(1+2 \mathbf{i}+4 \mathbf{j}+8 \mathbf{k})\left(2 U N_{2}^{(3)}-U N_{3}^{(3)}\right) \\
+(1-\mathbf{i}-\mathbf{j}+2 \mathbf{k})
\end{array}\right) .
\end{aligned}
$$

\section{Conclusions}

There are two differences between the dual third-order Jacobsthal and the dual coefficient third-order Jacobsthal quaternions. The first one is as follows: the dual coefficient third-order Jacobsthal quaternionic units are $\mathbf{i}^{2}=\mathbf{j}^{2}=\mathbf{k}^{2}=\mathbf{i j k}=-1$ whereas the dual third-order Jacobsthal quaternionic units are $\mathbf{i}^{2}=\mathbf{j}^{2}=\mathbf{k}^{2}=0, \mathbf{i j}=-\mathbf{j i}=\mathbf{j} \mathbf{k}=-\mathbf{k j}=\mathbf{k i}=-\mathbf{i k}=0$. The second one is as follows: the elements of the dual coefficient third-order Jacobsthal quaternion are $J_{m}^{(3)}+\varepsilon J_{m+1}^{(3)}\left(\varepsilon^{2}=0, \varepsilon \neq 0\right)$ whereas the elements of the dual third-order Jacobsthal quaternions are $m$-th third-order Jacobsthal number $J_{m}^{(3)}$.

\section{Acknowledgments}

The author would like to thank the anonymous referee for helpful comments on the original manuscript.

\section{References}

[1] E. Ata and Y. Yayl, Dual quaternions and dual projective spaces, Chaos, Solitons and Fractals, 40(3), pp. 1255-1263, (2009).

[2] P. Barry, Triangle geometry and Jacobsthal numbers, Irish Math. Soc. Bull. 51, pp. 45-57, (2003).

[3] G. Cerda-Morales, Identities for third order Jacobsthal quaternions, Advances in Applied Clifford Algebras 27 (2), pp. 1043-1053, (2017). 
[4] Ch. K. Cook and M. R. Bacon, Some identities for Jacobsthal and Jacobsthal-Lucas numbers satisfying higher order recurrence relations, Annales Mathematicae et Informaticae 41, pp. 27-39, (2013).

[5] Z. Ercan and S. Yüce, On properties of the dual quaternions,Eur. J. Pure Appl. Math. 4 (2), pp. 142-146, (2011).

[6] S. Halıcı, On Fibonacci quaternions, Advances in Applied Clifford Algebras 22(2), pp. 321-327, (2012).

[7] C. J. Harman, Complex Fibonacci numbers, The Fibonacci Quarterly. 19 (1), pp. 82-86, (1981).

[8] A. F. Horadam, A generalized Fibonacci sequence, The American Mathematical Monthly. 68 (5), pp. 455-459, (1961).

[9] A. F. Horadam, Complex Fibonacci numbers and Fibonacci quaternions, American Math. Monthly. 70 (3), pp. 289-291, (1963).

[10] A. F. Horadam, Quaternion recurrence relations, Ulam Quarterly. 2 (2), pp. 23-33, (1993).

[11] A. F. Horadam, Jacobsthal representation numbers, Fibonacci Quarterly 34 , pp. 40-54, (1996).

[12] A. L. Iakin, Generalized quaternions of higher order, The Fibonacci Quarterly. 15 (4), pp. 343-346, (1977).

[13] A. L. Iakin, Generalized quaternions with quaternion components, The Fibonacci Quarterly. 15 (4), pp. 350-352, (1977).

[14] M. R. Iyer, A note on Fibonacci quaternions, The Fibonacci Quarterly. 7 (3), pp. 225-229, (1969).

[15] M. R. Iyer, Some results on Fibonacci quaternions, The Fibonacci Quarterly. 7 (3), pp. 201-210, (1969).

[16] V. Majernik, Quaternion formulation of the Galilean space-time transformation, Acta Phy. Slovaca., 56 (1), pp. 9-14, (2006).

[17] K. S. Nurkan and I. A. Güven, Dual Fibonacci quaternions. Adv. Appl. Clifford Algebras. 25 (2), pp. 403-414, (2015).

[18] S. Yüce and F. Torunbalcı Aydın, A new aspect of dual Fibonacci quaternions, Adv. Appl. Clifford Algebras. 26 (2), pp. 873-884, (2016). 
[19] S. Yüce and F. Torunbalcı Aydın, Generalized dual Fibonacci quaternions, Applied Mathematics E-Notes 16, pp. 276-289, (2016).

\author{
Gamaliel Cerda-Morales \\ Instituto de Matemáticas, \\ Pontificia Universidad Católica de Valparaíso, \\ Blanco Viel 596, \\ Valparaíso, \\ Chile \\ e-mail: gamaliel.cerda.m@mail.pucv
}

